\title{
Extracción Rápida de Pectina a Partir de Cáscara de Maracuyá (Passiflora edulis flavicarpa) empleando Microondas
}

\author{
Katty J. Urango-Anaya, Fabián A. Ortega-Quintana, Gabriel Vélez-Hernández* y Ómar A. Pérez-Sierra \\ Universidad de Córdoba, Facultad de Ingenierías, Programa de Ingeniería de Alimentos, Grupo de \\ investigación GIPPAL, Carrera 6 No. 76-103, Montería - Córdoba, Colombia. \\ (e-mail: gvelez@correo.unicordoba.edu.co) \\ ${ }^{*}$ Autor a quien debe ser dirigida la correspondencia
}

Recibido Abr. 20, 2017; Aceptado Jun. 28, 2017; Versión final Ago. 31, 2017, Publicado Feb. 2018

\begin{abstract}
Resumen
La presente investigación muestra los resultados de rendimiento del proceso de extracción de pectina de la cáscara del fruto de maracuyá (Passiflora edulis flavicarpa) asistida por microondas y el contenido de metoxilo de la pectina obtenida. Se usó un diseño completamente aleatorizado con arreglo factorial $3^{3}$ por triplicado, con los siguientes factores: tiempo de extracción, potencia del microondas y concentración de la solución de $\mathrm{HCl}$. Se realizó un análisis de varianza y un test de comparación de medias con un nivel de significancia del $5 \%$. Las condiciones que arrojaron el mayor rendimiento en pectina $(68,76 \%)$ en base húmeda fueron: tiempo de 100 segundos, potencia de 1000 vatios y concentración de solución de $\mathrm{HCl}$ de $0,24 \mathrm{~N}$. Para este tratamiento, el porcentaje del contenido de metoxilo de la pectina obtenida fue del $6,86 \%$ $(\mathrm{p} / \mathrm{p})$, clasificando a la pectina como de bajo metoxilo.
\end{abstract}

Palabras clave: pectina, metoxilo, extracción, microonda, maracuyá

\section{Rapid Extraction of Pectin from Passion Fruit Peel (Passiflora edulis flavicarpa) using Microwave}

\begin{abstract}
In this research, the performance of the microwave-assisted extraction process of pectin from peel of passion fruit (Passiflora edulis flavicarpa) and the methoxyl content of the pectin obtained are shown. A completely randomized design with factorial arrangement $3^{3}$ in triplicate, was employed. The following factors were considered: extraction time, microwave power and concentration of the solution of $\mathrm{HCl}$. Analysis of variance (ANOVA) was performed and a mean comparison test with a significance level of $5 \%$ was applied. The results show that the treatment with the highest pectin yield $(68,76 \%)$, in wet basis, had the following conditions: time of 100 seconds, power of $1000 \mathrm{~W}$ and concentration of $\mathrm{HCl}$ of $0,24 \mathrm{~N}$. For this treatment, the percentage of the methoxyl content of the pectin obtained was $6,86 \%(\mathrm{w} / \mathrm{w})$, classifying the pectin as low methoxyl.
\end{abstract}

Keywords: pectin, methoxyl, extraction, microwave, passion fruit 


\section{INTRODUCCIÓN}

La Maracuyá (Passiflora edulis flavicarpa) es una planta enredadera que ha sido introducida y cultivada con gran interés comercial en la mayoría de las regiones tropicales y subtropicales del planeta. Es altamente apreciada por su fruto debido a los beneficios que proporciona y gracias a su contenido de pectina es empleado en la industria alimenticia e industria farmacéutica (Fishman et al., 1999; Kulkarni et al., 2010; Sharma et al., 2014). Tradicionalmente la pectina ha sido extraída de frutas cítricas y de pulpa de manzana (Wang et al., 2007), pero debido a la considerable producción de maracuyá en Colombia (80.000 ton/año) (Legiscomex, 2013), se busca una alternativa de los subproductos que ellos generan, como lo es la cáscara, que es de alrededor de $50 \%$ a $60 \%$ del fruto, una de esta alternativa es obtener pectina y así disminuir el impacto ambiental que originan los desperdicios industriales.

La pectina es un polisacárido que absorbe gran cantidad de agua y es un producto tecnológicamente funcional de interés para la industria de alimentos en el desarrollo de productos debido a que sus propiedades reológicas son apropiadas para la elaboración de jaleas, mermeladas, salsas, entre otros, aportando así a la textura y consistencia (D’Addosio et al., 2005; Seixas et al., 2014). Debido a la gran importancia industrial de la pectina, se requieren métodos con mayor rendimiento de extracción y que den como resultado un producto de buena calidad. Algunas características importantes que definen la calidad de las pectinas son (Liew et al., 2014): 1) El grado de metoxilación y 2) El poder gelificante.

El grado de metoxilación (o contenido de metoxilo), el cual se refiere a la esterificación de los grupos carboxilos por radicales metilos. Basado en este grado de metoxilación, se distinguen dos tipos de pectina: i) Pectinas de Alto Metoxilo (PAM), en estas pectinas más del $50 \%$ de los grupos carboxilo del ácido galacturónico del polímero se encuentran esterificados con metanol. Las pectinas de alto metoxilo se subdividen en dos grupos: pectinas de gelificación rápida, cuyo tiempo de gelificación es menor a cinco minutos y un grado de esterificación con metanol entre 68 y $75 \%$, y las pectinas de gelificación lenta, cuyo tiempo de gelificación es mayor de cinco minutos y un grado de esterificación con metanol entre 60 y $68 \%$. ii) Pectinas de Bajo Metoxilo (PBM): en estas pectinas, menos del $50 \%$ de los grupos hidroxilo están esterificadas con metanol. En la gelificación, estas pectinas requieren la presencia de cationes divalentes como el calcio. Una pectina puede tener un contenido de metoxilo alrededor del $14 \%$. De aquí que se ha fijado el $7 \%$ de contenido de metoxilo (50\% de esterificación con metanol) como el límite para diferenciar las categorías de pectina sobre la base del contenido de metoxilo (Liu et al., 2010). El poder gelificante, definido como la capacidad que tiene una pectina para formar gel. Esta capacidad depende de la molécula péctica y de su grado de metoxilación (Badui, 2013).

Para la extracción de pectinas se han empleados diversas técnicas: las convencionales como la extracción por arrastre de vapor e hidrodestilación, las no convencionales como la extracción asistida por microondas (EAM) y la extracción por hidrólisis ácida que es aplicada ampliamente a nivel industrial (Qiu et al., 2010). La extracción asistida por microondas es un método recientemente utilizado con el propósito de liberar la pectina a partir de diversas materias primas y con su utilización se han encontrado resultados bastante deseables en el proceso de extracción de la pectina como la reducción de los tiempos de extracción, altos rendimientos en pectina y buena calidad del producto obtenido (López et al., 2011). La técnica de extracción asistida por microondas se basa en el uso de la energía de microondas para conseguir que los compuestos de interés pasen rápidamente de la muestra a un disolvente adecuado, además, es una técnica que utiliza volúmenes pequeños de solvente y permite el control de una serie de parámetros que afectan a la eficacia de extracción (Seixas et al., 2014).

Para el caso de la extracción de pectina de la cáscara de maracuyá se han realizado investigaciones con los métodos convencionales e hidrólisis ácida, obteniéndose rendimientos de extracción entre 10 y el $70 \%$ (Kliemann et al., 2009; Liew et al., 2014; Freitas de Oliveira et al., 2016), estudios realizados por Kalapathy y Proctor (2000) y Seggiani et al., (2009) indican que la extracción con $\mathrm{HCl}$ se obtienen mayores rendimientos y mejor calidad de pectina y menor consumo de alcohol en la precipitación y Aunque existen reportes de extracción de pectina de la cáscara de maracuyá asistida por microondas, estos no tienen en cuenta la variación de la concentración del ácido utilizado, el cual ofrece un gran aporte en la hidrólisis de la protopectina y exposición de la pectina al solvente (Seixas et al., 2014).

Por lo tanto, el objetivo de este trabajo fue evaluar el efecto del tiempo de extracción, la concentración de ácido clorhídrico y la potencia del microondas sobre el rendimiento de la pectina extraída a partir de cáscaras del fruto de maracuyá (Passiflora edulis flavicarpa) utilizando la técnica de extracción asistida por microondas. 


\section{METODOLOGÍA}

La metodología se presenta en cuatro subsecciones: en la primera se describe cómo se seleccionó la materia prima, la segunda describe el proceso de extracción utilizado, la tercera el análisis realizado a la pectina y la última describe el diseño experimental.

\section{Materia prima}

Se utilizó como materia prima la cáscara del fruto de maracuyá (Passiflora edulis flavicarpa) adquirida en el mercado local de Cereté, Colombia, seleccionadas en estado de madurez 5 , según la metodología descrita por Torres et al. (2013).

\section{Equipo y procedimiento de extracción de pectina}

La figura 1 muestra el montaje utilizado para la extracción de la pectina y en la figura 2 se describe el procedimiento utilizado para la extracción de pectina.

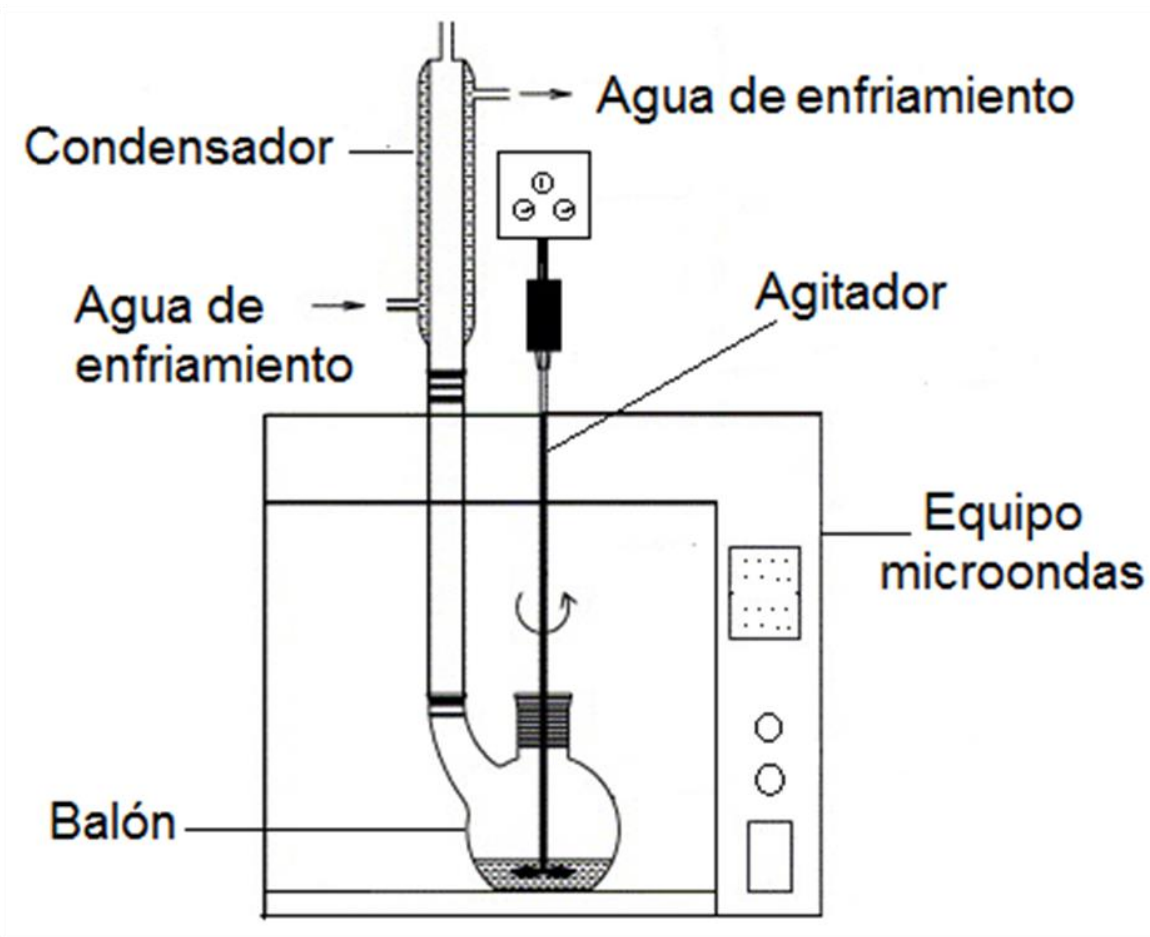

Fig. 1: Esquema del equipo utilizado para la extracción de pectina (Adaptado de Wang et al., 2007).

\section{Calculo del rendimiento de pectina}

Para la determinación del rendimiento de pectina fue utilizada la ecuación (1)

Rendimiento $(\%)=$ Pectina húmeda $(\mathrm{g}) /$ Muestra inicial de cáscara $(\mathrm{g})$ *100

\section{Determinación del contenido de Metoxilo de la pectina.}

El contenido de metoxilo se determinó a partir de la titulación, con $\mathrm{NaOH} 0,1 \mathrm{~N}$ y fenolftaleína como indicador, de $2 \mathrm{~g}$ de pectina seca extraída y preparada por la metodología descrita en Singthong et al. (2004).

\section{Diseño Experimental}

Para el análisis del efecto del tiempo de extracción, potencia del microondas y concentración de la solución de ácido $(\mathrm{HCl})$ sobre el rendimiento de la pectina (\% en base húmeda) se empleó un diseño completamente aleatorizado (DCA) con arreglo factorial $3^{3}$ con el tiempo de hidrólisis de $\{60,80,100\}$ segundos, la potencia del microondas de $\{600,800,1000\}$ vatios y la concentración de la solución de $\mathrm{HCl}$ de $\{0,12 ; 0,18 ; 0,24\} \mathrm{N}$. Para el análisis estadístico se realizó un análisis de varianza (ANOVA) con un nivel de significancia de $5 \%$ utilizando Statgraphics Centurion XV y se aplicó prueba de comparación de medias de Duncan. 


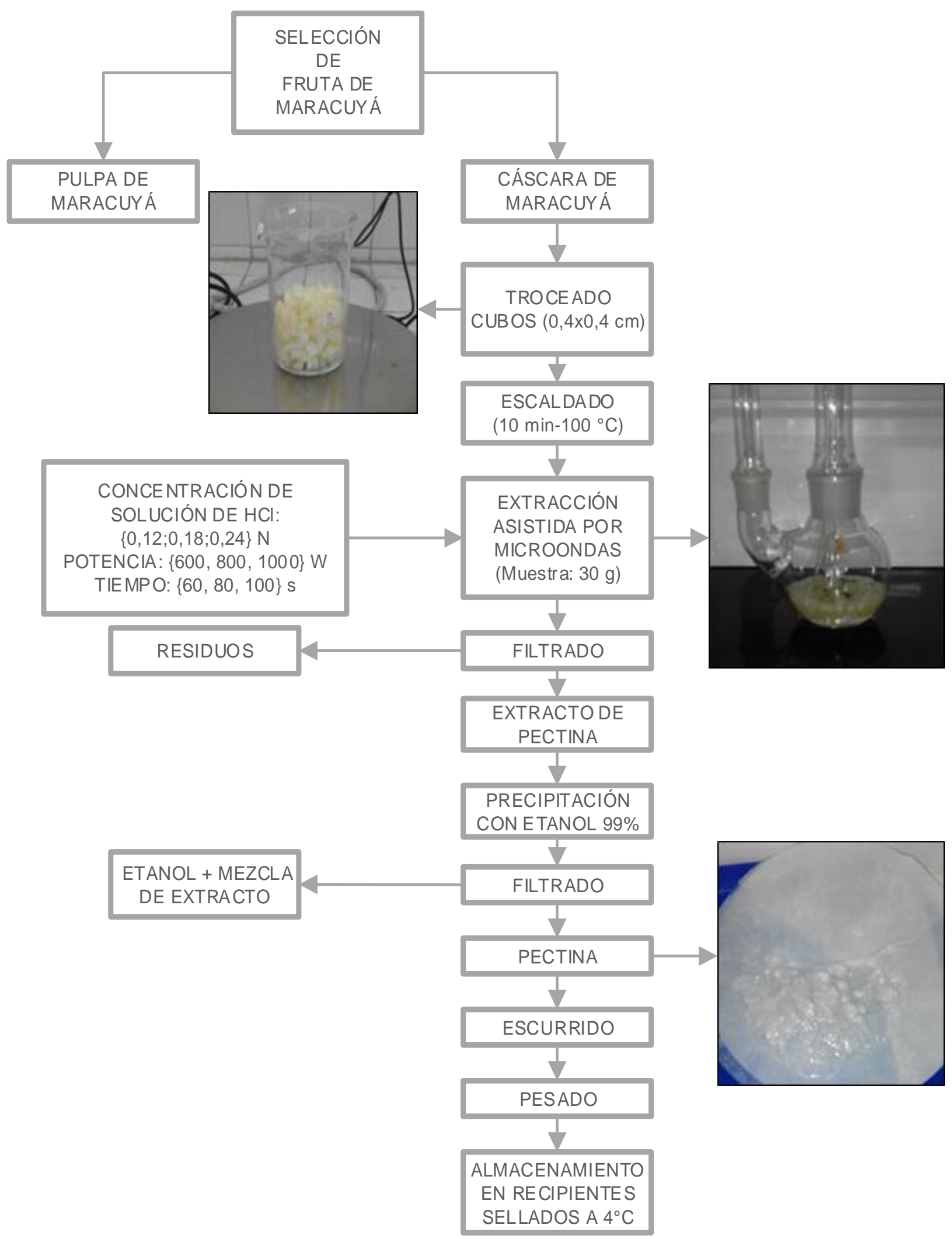

Fig. 2: Esquema del proceso de extracción de pectina.

\section{RESULTADOS Y DISCUSIÓN}

En la tabla 1 se presenta el análisis de varianza (ANOVA) para los datos experimentales de rendimiento de pectina de la cáscara del fruto de maracuyá. Debido a que todos los valores $p$ son inferiores a 0,05 , la concentración de solución de ácido clorhídrico, el tiempo de hidrólisis o extracción, la potencia del microondas y sus interacciones tuvieron efecto estadísticamente significativo sobre el rendimiento de pectina de la cáscara del fruto de maracuyá. 
Tabla 1: Análisis de varianza para el porcentaje de rendimiento de la pectina. A: concentración de la solución ácida de $\mathrm{HCl}(\mathrm{N})$, B: tiempo (s), C: potencia (W), GL: Grados de libertad

\begin{tabular}{|l|c|c|c|c|c|}
\hline Fuente & GL & Suma de Cuadrados & Cuadrado medio & Valor $F$ & $p>F$ \\
\hline $\mathrm{A}$ & 2 & 2221,53 & 1110,76 & 1624,20 & $<0,0001$ \\
\hline $\mathrm{B}$ & 2 & 789,51 & 394,75 & 577,22 & $<0,0001$ \\
\hline $\mathrm{C}$ & 2 & 285,36 & 142,68 & 208,63 & $<0,0001$ \\
\hline $\mathrm{A}^{*} \mathrm{~B}$ & 4 & 69,84 & 17,46 & 25,53 & $<0,0001$ \\
\hline $\mathrm{A}^{*} \mathrm{C}$ & 4 & 14,61 & 3,65 & 5,34 & 0,0011 \\
\hline $\mathrm{B}^{*} \mathrm{C}$ & 4 & 61,96 & 15,49 & 22,65 & 0,0011 \\
\hline $\mathrm{A}^{*} \mathrm{~B}^{*} \mathrm{C}$ & 8 & 43,81 & 5,48 & 8,01 & 0,0011 \\
\hline
\end{tabular}

\section{Extracción de pectina}

La tabla 2 muestra los porcentajes promedios de rendimiento obtenidos para los diferentes tratamientos aplicados a la cáscara de maracuyá y la figura 3 muestra las tendencias de los rendimientos para cada tiempo de extracción con respecto a la potencia del microondas y concentración de solución de $\mathrm{HCl}$. La prueba de comparación de medias de Duncan $(p<0,05)$, reportada en la tabla 2, muestra que el tratamiento que presentó el rendimiento en pectina más alto fue un tiempo de hidrólisis de 100 segundos con concentración $0,24 \mathrm{~N}$ de la solución de $\mathrm{HCl}$ y una potencia de 1000 vatios. En la figura 3 se observa que el rendimiento en pectina, para cada tiempo de extracción, es mayor cuando aumentan la potencia del microondas y la concentración de $\mathrm{HCl}$. Al respecto, Seixas et al. (2014) explican que el daño del tejido vegetal incrementa cuando incrementa la intensidad del campo o potencia del microondas, lo cual es evidenciado por un incremento en la porosidad capilar de la cáscara, así que la célula se dividirá y la pectina puede liberarse.

Por otro lado, Cuesta y Muñoz (2012) utilizaron la técnica de hidrólisis ácida con $\mathrm{HCl}$ para la extracción de pectina a partir de cáscara de maracuyá a diferentes valores de $\mathrm{pH}(2,3$ y 4), encontrando que a mayores concentraciones de ácido el porcentaje de pectina extraída es mayor debido a la presencia de altas concentraciones de $\mathrm{H}^{+}$que aporta el ácido y que hidroliza a la protopectina dejando así expuesta la pectina al solvente de extracción. La protopectina es un compuesto formado por la combinación de celulosa con moléculas de pectina y cuando la concentración de $\mathrm{H}^{+}$de la solución incrementa, el grupo carboxilado hidratado es convertido a grupos de ácido carboxilo hidratados, el contenido bajo de grupos carboxilados es capaz de reducir la repulsión de las moléculas de polisacárido lo cual promueve el aumento de la cantidad de precipitado de pectina (Liew et al., 2014)

Adicionalmente, Sánchez et al. (2011) encontraron que dentro de los métodos de extracción tales como: extracción asistida por microondas, convencional, enzimática y fisicoquímicas, resulta más eficiente el método asistido por microondas en cuanto a rendimiento, calidad de la pectina y tiempos de extracción. Por lo que proponen el empleo de esta técnica para la obtención de pectinas a partir de frutas. Esto se debe al rápido calentamiento producido por la energía del campo electromagnético al convertirse en calor cuando interactúan con las moléculas polares, como el agua, causando formación de vapores intensos dentro de la estructura porosa del material vegetal, lo cual produce ablandamiento e hidrólisis del tejido orgánico de la cáscara disminuyendo así los tiempos de extracción de la pectina (Kratchanova et al., 2004; Wang et al., 2007; ReyesGarcía et al., 2010).

Tabla 2: Rendimiento en pectina de la cáscara del fruto de maracuyá.

\begin{tabular}{|c|c|c|c|c|}
\hline \multirow{2}{*}{$\begin{array}{c}\text { Concentración de } \\
\mathrm{HCl}(\mathrm{N})\end{array}$} & $\begin{array}{c}\text { Potencia } \\
\text { (Vatios) }\end{array}$ & \multicolumn{3}{|c|}{$\begin{array}{c}\text { Rendimiento en pectina (\%p/p) } \\
\pm \text { desviación estándar }\end{array}$} \\
\cline { 2 - 5 } & & $t=60 \mathrm{~s}$ & $t=80 \mathrm{~s}$ & $t=100 \mathrm{~s}$ \\
\hline \multirow{2}{*}{0,12} & 600 & $47,94^{\mathrm{a}} \pm 0,54$ & $54,47^{\mathrm{e}} \pm 0,73$ & $59,07^{\mathrm{g}} \pm 0,44$ \\
\cline { 2 - 5 } & 800 & $48,80^{\mathrm{b}} \pm 0,61$ & $56,57^{\mathrm{f}} \pm 0,77$ & $59,86^{\mathrm{g}} \pm 0,38$ \\
\cline { 2 - 5 } & 1000 & $49,23^{\mathrm{c}} \pm 0,39$ & $58,22^{\mathrm{fg}} \pm 0,43$ & $61,02^{\mathrm{gh}^{\mathrm{h}} \pm 1,22}$ \\
\hline \multirow{2}{*}{0,18} & 600 & $49,30^{\mathrm{c}} \pm 0,28$ & $63,48^{\mathrm{h}} \pm 0,24$ & $63,64^{\mathrm{i}} \pm 0,61$ \\
\cline { 2 - 5 } & 800 & $53,34^{\mathrm{d}} \pm 0,96$ & $64,79^{\mathrm{i}} \pm 0,34$ & $66,52^{\mathrm{k}} \pm 0,80$ \\
\cline { 2 - 5 } & 1000 & $54,45^{\mathrm{e}} \pm 0,28$ & $65,91^{\mathrm{j}} \pm 2,61$ & $67,94^{\mathrm{k}} \pm 0,86$ \\
\hline \multirow{2}{*}{0,24} & 600 & $50,75^{\mathrm{c}} \pm 0,22$ & $57,52^{\mathrm{f}} \pm 0,86$ & $65,79^{\mathrm{ik}} \pm 0,91$ \\
\cline { 2 - 5 } & 800 & $56,11^{\mathrm{f}} \pm 0,79$ & $64,97^{\mathrm{i}} \pm 0,39$ & $68,41^{\mathrm{k}} \pm 0,84$ \\
\cline { 2 - 5 } & 1000 & $60,00^{\mathrm{g}} \pm 0,33$ & $66,93^{\mathrm{k}} \pm 0,34$ & $68,73^{\mathrm{m}} \pm 0,30^{*}$ \\
\hline
\end{tabular}

*Tratamiento que arrojó mayor rendimiento de pectina; los superíndices ${ }^{\mathrm{a}-\mathrm{m}}$ indican diferencia significativa 
Con la técnica de microondas utilizada en el presente trabajo de investigación se estudió el rendimiento de la pectina con tiempo límite de 100 segundos, donde los valores obtenidos de rendimientos en pectina oscilan entre el 47,94 a $68,73 \%$ p/p (en base húmeda). Estos rendimientos son mayores comparados a lo reportado por otras investigaciones con otros métodos de extracción en las cuales los tiempos de extracción son bastante altos lo cual produce disminución en la calidad de la pectina debido a su deterioro (Kliemann et al., 2009; Liew et al., 2014).
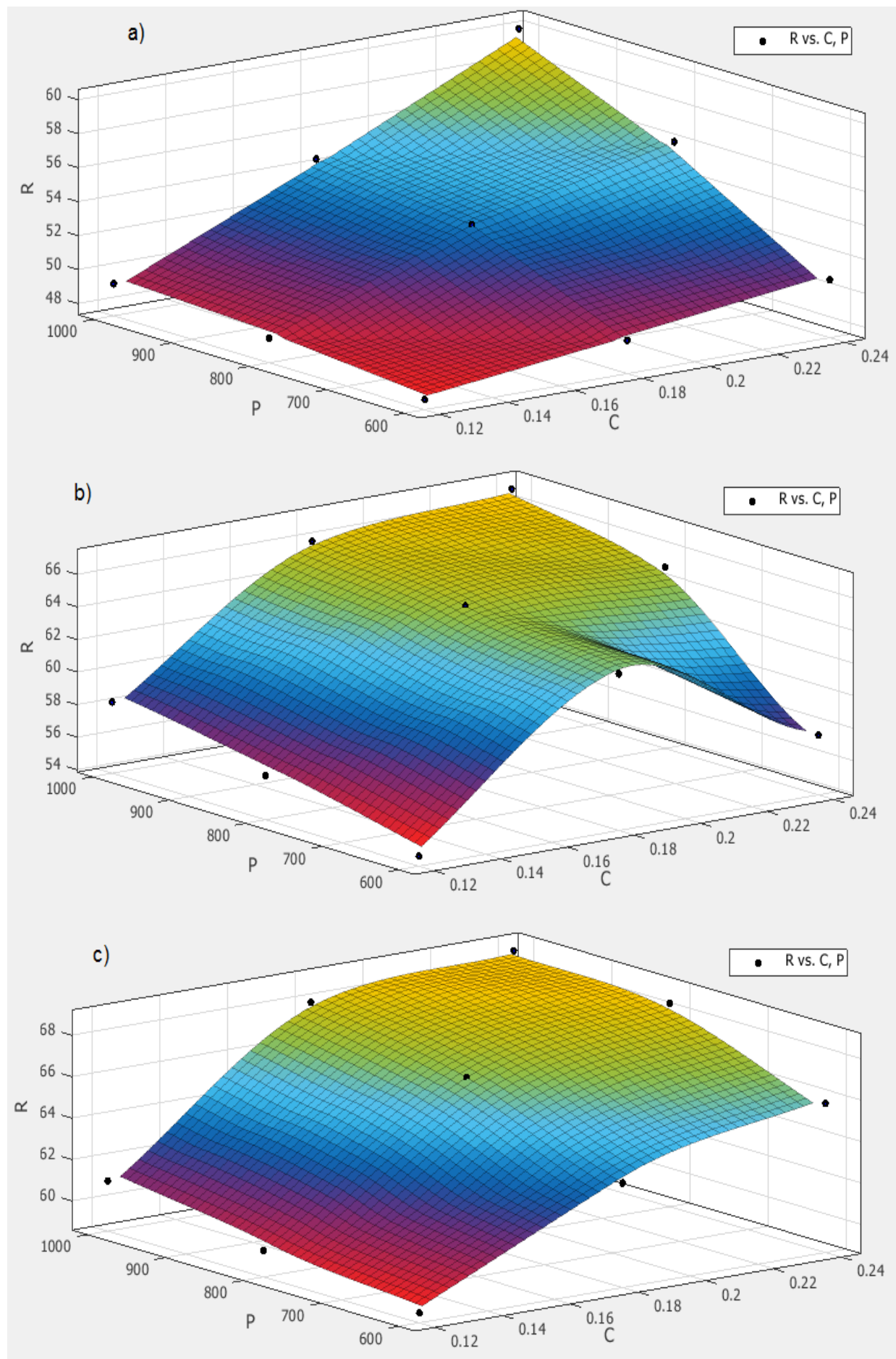

Fig. 3: Rendimiento en pectina (\%p/p) a diferentes tiempos de extracción: a) $60 \mathrm{~s}, \mathrm{~b}) 80 \mathrm{~s}, \mathrm{c}) 100 \mathrm{~s}$. (P: potencia, $\mathrm{C}$ : concentración del ácido, R: porcentaje de rendimiento en pectina). (Los puntos indican datos experimentales).

\section{Caracterización de la pectina extraída}

Adicional a los rendimientos altos de pectina, también se tuvo en cuenta la calidad de la pectina obtenida. Para esto, se tomó como parámetro de evaluación el contenido de metoxilos (expresado en porcentaje) del tratamiento que arrojó un mayor rendimiento, es decir, para un tiempo de hidrólisis de 100 segundos, 
concentración de la solución de $\mathrm{HCl}$ de $0,24 \mathrm{~N}$ y potencia del microondas de 1000 vatios. El contenido de metoxilos para este tratamiento fue de $6,86 \pm 0,5 \%(\mathrm{p} / \mathrm{p})$, como este valor es menor al valor límite de referencia correspondiente al $7 \%$ de metoxilo, la pectina obtenida fue clasificada como pectina de bajo metoxilo. Este valor bajo de contenido de metoxilo es causado por la descomposición de la estructura de la pectina causada por el ácido (Cuesta y Muñoz, 2010). Cuesta y Muñoz (2010) demostraron que la concentración de ácido $(\mathrm{HCl})$ de la solución para la extracción de pectinas a partir de cáscara de maracuyá con valores de pH alrededor de 2 se obtiene un mayor rendimiento de la pectina, pero esta no presentó un contenido alto de metoxilo, en cambio para valores de $\mathrm{pH}$ mayores a 4, la pectina obtenida con menor rendimiento era de mejor calidad, con contenidos de metoxilos mayores del $13 \%$.

\section{CONCLUSIONES}

La extracción de pectina asistida por microondas se observó que la concentración de solución de ácido clorhídrico, el tiempo de hidrólisis o de extracción, la potencia del microondas y las interacciones de estas tres variables tuvieron efecto estadísticamente significativo $(p<0,05)$ sobre el rendimiento de pectina de la cáscara del fruto de maracuyá.

En el proceso de extracción de pectina a partir de cáscaras del fruto de maracuyá asistida por microondas se obtuvo un mayor rendimiento para el tratamiento con tiempo de 100 segundos, potencia de 1000 vatios y concentración de la solución ácida $(\mathrm{HCl})$ de $0,24 \mathrm{~N}$ y el porcentaje de pectina en base húmeda obtenida a estas condiciones fue del $68,73 \%(p / p)$. El porcentaje del contenido de metoxilos de la pectina obtenida fue del $6,86 \%$, este resultado la clasifica como pectina de bajo metoxilo.

\section{REFERENCIAS}

Badui, S., Química de los Alimentos, 5ª edición, 78-81. Pearson, Ciudad de México, México (2013)

Cuesta, M. y R. Muñoz, Extracción de pectina a partir de la corteza de maracuyá (Passiflora edulis var. Flavicarpa Degener). Rev. Politécnica, 31(1), 91-96 (2010)

D’Addosio, R.; G. Páez; M. Marín; Z. Mármol y J. Ferrer, Obtención y caracterización de pectina a partir de la cáscara de parchita (Passiflora edulis f. flavicarpa Degener). Rev. Fac. Agron., 22(3), 241-251 (2005)

Fishman, M., y otros tres autores, Characterization of pectin, flash-extracted from orange albedo by microwave heating, under pressure. Carbohydrate Research, 323, 126-38 (1999)

Freitas de Oliveira, F., y otros cinco autores, Extraction of pectin from passion fruit peel assisted by ultrasound. LWT-Food Science and Technology, 71, 110-115 (2016)

Kalapathy, U. y Proctor, A., Effect of acid extraction and alcohol precipitation conditions on the yield and purity of soy hull pectin. Food Chemistry, 73 (4), 393-396 (2001)

Kliemann, E., y otros seis autores, Optimisation of pectin acid extraction from passion fruit peel (Passiflora edulis flavicarpa) using response surface methodology. International Journal of Food Science and Technology, 44(3), 476-483 (2009)

Kratchanova, M.; Pavlova, E. y Panchev, I., The effect of microwave heating of fresh orange peels on the fruit tissue and quality of extracted pectin. Carbohydrate Polymers, 56, 181-185 (2004)

Kulkarni, S. y Vijayanand, P., Effect of extraction conditions on the quality characteristics of pectin from passion fruit peel (Passiflora edulis $f$. flavicarpa L.). LWT - Food Science and Technology, 43(7), 1026-1031 (2010)

Legiscomex. Inteligencia de mercados / Exportación de frutas exóticas colombianas (en la web: https://goo.gl/q1MMKb, acceso 4 de mayo de 2016), LEGIS S.A., Colombia (2013)

Liew, S.; Chin, N. y Yusof, Y., Extraction and Characterization of Pectin from Passion Fruit Peels. Agriculture and Agricultural Science Procedia, 2, 231-236 (2014)

López, N., y otros cuatro autores, Pectina de mango: perspectivas para su extracción, Cienciacierta, 7(27) (2011)

Liu, L., y otros cuatro autores, Extraction of pectins with different degrees of esterification from mulberry branch bark. Bioresource Technology, 1001(9), 3268-3273 (2010) 
Qiu, L., y otros cinco autores, Investigation of combined effects of independent variables on extraction of pectin from banana peel using response surface methodology. Carbohydrate Polymers, 80(2), 326-331 (2010)

Reyes-García, V.; López-Malo, A; y Sosa-Morales, M., Efectos del calentamiento con microondas en alimentos fluidos. Temas Selectos de Ingeniería de Alimentos, 4(2), 38-47 (2010)

Sanchez, D., y otros tres autores, Moléculas pécticas: extracción y su potencial aplicación como empaque. Tecnociencia Chihuahua, V (2), 76-82 (2011)

Seixas, F., y otros seis autores, Extraction of pectin from passion fruit peel (Passiflora edulis $f$. flavicarpa) by microwave-induced heating. Food Hydrocolloids, 38, 186-192 (2014)

Seggiani, M., y otros cuatro autores, Effect of different extraction and precipitation methods on yield and quality of pectin. International Journal of Science and Technology, 44, 574-580 (2009)

Sharma, P.; Gupta, A. y Kaushal, P., Optimization of method for extraction of pectin from apple pomace. Indian Journal of Natural Products and Resources, 5(2), 184-189 (2014)

Singthong, J., y otros tres autores, Structural characterization degree of esterification and some gelling properties of Krueo Ma Noy (Cissampelos pareira) pectin. Carbohydrate polymers, 58, 391-400 (2004)

Torres, R., y otros tres autores, Relación del color y del estado del madurez con las propiedades fisicoquímicas de frutas tropicales. Información Tecnologíca, 24(3), 51-56 (2013)

Wang, S., y otros cinco autores, Optimization of pectin extraction assisted by microwave from apple pomace using response surface methodology. Journal of Food Engineering, 78(2), 693-700 (2007) 